\title{
The influence of Cr concentration on time resolution of GaAs detectors
}

\author{
L.L. Fedorenko ${ }^{1}$, L.F. Linnik ${ }^{1}$, L.G. Linnik ${ }^{1}$, M.M. Yusupov ${ }^{1}$, E.A. Solovyov ${ }^{1}$, E. Sirmulis ${ }^{2}$ \\ ${ }^{I} V$. Lashkaryov Institute of Semiconductor Physics, NAS of Ukraine, \\ 45, prospect Nauky, 03028 Kyiv, Ukraine \\ Phone: +380 (44) 525-64-77, +380 (44) 525-18-75, +380 (44) 525-59-39; \\ e-mail:lfedor@isp.kiev.ua,ny@isp.kiev.ua,slv@isp.kiev.ua \\ ${ }^{2}$ Semiconductor Physics Institute, 11, Gostauto, Vilnius Lt-01108, Lithuania; \\ E-mail: sirmulis@delfi.lt
}

\begin{abstract}
Investigated in this work were the influence of $\mathrm{Cr}$ dopant concentration and technological conditions of doping on photoconductivity $(\mathrm{PhC})$ kinetics, dependence of $\mathrm{PhC}$ signal magnitude on voltage applied as well as the dynamic range of a photodetector based on semi-insulating GaAs:Cr. PhC relaxation was measured using a broadband system of registration in the picosecond pulse range, which is based on the oscillograph C7-19, CCD camera and personal computer. Mechanisms of recombination that influence on fast and slow components of the $\mathrm{PhC}$ signal were studied. The shortest time of $\mathrm{PhC}$ relaxation $\tau \sim 2 \cdot 10^{-10} \mathrm{~s}$ was observed in GaAs: $\mathrm{Cr}$ samples for the chromium dopant concentration $N_{\mathrm{Cr}} \sim 3 \cdot 10^{17} \mathrm{~cm}^{-3}$. We have found a linear increase of the fast component of $\mathrm{PhC}$ with the intensity of excitation as well as a weak dependence at small levels and saturation at the high ones of excitation for the $\mathrm{PhC}$ slow component.
\end{abstract}

Keywords: GaAs, fast detector, picosecond YAG-laser, X-, $\gamma$-radiation, photoconductivity.

Manuscript received 07.02.06; accepted for publication 29.03.06.

\section{Introduction}

Diagnostic of the pulse radiation process requires the development of ultra-fast X-ray, $\gamma$ - and laser radiation detectors with nano- and picosecond times of relaxation. The detectors must precisely reproduce a pulse shape and measure its magnitude with well linearity within few orders. For such a purpose, used are semiconductor compounds with a high mobility of intrinsic charge carriers and high sensitivity to X-ray and $\gamma$-radiation, e.g. GaAs, InPCdTe, CdZnTe $[1,2]$. Subnanosecond time resolution can be realized using detectors based on high resistive GaAs, InP $[3,4]$. A short drop of relaxation can be obtained by decreasing the lifetime of photocarrier, e.g., $\mathrm{Cr}$ doping, neutron irradiation, or low temperature growing GaAs [5]. The best semi-insulating GaAs was grown with $\mathrm{Cr}$ doping concentration $N_{\mathrm{Cr}} \sim 1 \cdot 10^{16} \mathrm{~cm}^{-3}$ and background impurities concentration $N_{\mathrm{ph}} \leq$ $\leq 5 \cdot 10^{15} \mathrm{~cm}^{-3}$. Nevertheless, a short relaxation drop of the photoresponse usually takes place in the long-term component. The latter could be conditioned by the influence of both type charge carriers sticking to shallow centers, bending of the energy band in semi-insulating GaAs, low hole mobility or carrier diffusion in the field range. According to the well-known relation

$\Delta \sigma \sim e \mu \Delta n$,

where $\Delta n=\beta k I \tau, \Delta \sigma$ - detector photoconductivity, $\Delta n$ - concentration, $\mu-$ mobility, $\tau$ - lifetime of a photocarrier; $\Delta \sigma$ decreases with $\tau$. For the pulse shape to be reproduced, the criterion $\tau<<\tau_{r}$ (where $\tau_{r}$ is the characteristic time of process relaxation) should be satisfied. At the same time, the magnitude of $\tau$ must be sufficient to adjust the detector sensitivity. The aim of the paper was to determine the optimal degree of crystal doping for fast $(\tau \leq 200 \mathrm{ps})$ and highly sensitivity detectors based on GaAs doped with chromium.

\section{Experimental}

To achieve the purpose mentioned above, the set-up for photoconductivity $(\mathrm{PhC})$ kinetics measurements in semiconductor crystals under pulse laser excitation was 
designed and adjust. The block scheme of the set-up is given in Fig. 1. The set-up consists of the YAG: $\mathrm{Nd}^{+3}$ laser with free mode-locking (1), microstrip circuit of photoconductivity signal detection (2), broad-band oscillograph (3), CCD camera (4), and PC with frame grabber (5). The train of picosecond pulses with the duration $t_{p}=37 \mathrm{ps} \quad\left(t_{p}\right.$ being much less than the characteristic nonstationary relaxation time $\tau_{\text {nst }}$ ) and $\sim 16$ ns pulse intervals was obtained using passive solidstate shutter based on polystyrene dye. The synchronization scheme of a trigger laser with oscillograph scanning and trigger of frame grabber was developed. We used semi-insulating GaAs samples with the various Cr concentrations $N_{\mathrm{Cr}} \sim 1 \cdot 10^{15} \ldots 3 \cdot 10^{17} \mathrm{~cm}^{-3}$, which were grown by the Czochralsky technique: using fluxing agent and horizontally oriented crystallization. To provide compensation, the GaAs: Cr crystals were grown in As vapor that ensure an electron type of conductivity when $\rho \sim 10^{9} \mathrm{Ohm} \mathrm{cm}$. The influence of the $\mathrm{Cr}$ dopant concentration on photoconductivity drop, the dependence of the $\mathrm{PhC}$ signal magnitude on the voltage applied and the dependence of the dynamic range of GaAs: $\mathrm{Cr}$ photodetectors on the radiation intensity were investigated.

The samples possessed the bar-like shape with dimensions $2 \times 2 \times 5 \mathrm{~mm}$. Their ohmic contacts were formed on the longer opposite sides, see insert in Fig. 1. Conductors had minimal length and were made of thin cooper foil $\delta \leq 10 \mu \mathrm{m}$ to reduce parasitic reactive resistance and skin-effect conjunctive. The samples were mounted in specially developed microstrip circuit adjusted with the impedance $\rho=50 \mathrm{Ohm}$.

Typical oscillograms of GaAs: $\mathrm{Cr} \mathrm{PhC}$ are shown in Fig. 2. Fig. 3 shows the dependence of GaAs photodetector signal magnitude on the voltage applied.

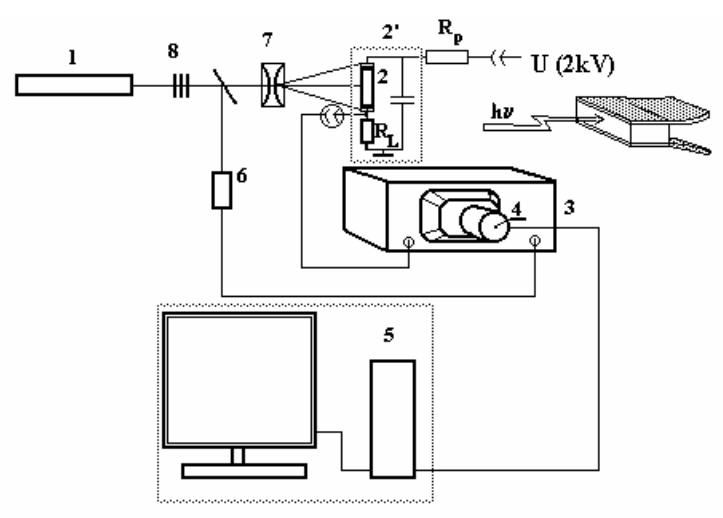

Fig. 1. Block-scheme of the pulse $\mathrm{PhC}$ registration set-up with the subnanosecond duration of excitation: 1 - laser with freemode locking, 2 - detecting semiconductor element, 3 oscillograph C7-19, 4 - CCD camera GBC 500E, 5 - PC with video card Rage Fury Pro 32M, 6 - photodiode FD-256, 7 lens, $\mathrm{NG}$ - filters.
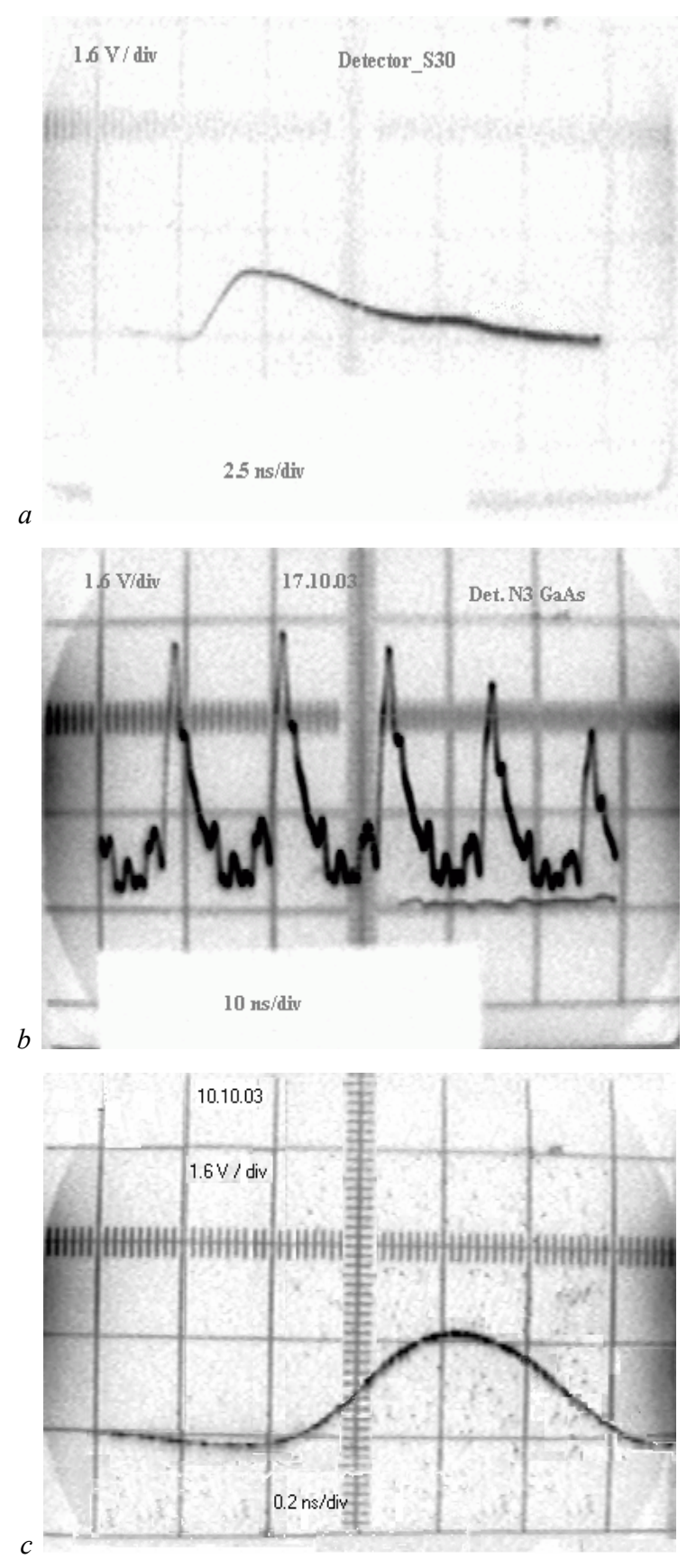

Fig. 2. Typical oscillograms of $\mathrm{PhC}$ pulses of GaAs samples with the $\mathrm{Cr}$ concentration $N_{\mathrm{Cr}}: \sim 110^{15} \mathrm{~cm}^{-3} \quad(\mathrm{a}, \mathrm{b})$; $\sim 3 \cdot 10^{17} \mathrm{~cm}^{-3}$ (c, d).

\section{Results and discussion}

We found that, with increasing the dopant concentration $N_{\mathrm{Cr}}$, the magnitude of the long-term component of $\mathrm{PhC}$ relaxation is decreased, which is indicative of the extension of the fast recombination channel contribution. The highest rate $\tau_{\mathrm{st}} / \tau_{\mathrm{nst}}>10\left(\tau_{\mathrm{st}}-\right.$ stationary relaxation time) was observed at $N_{\mathrm{Cr}} \cong 3 \cdot 10^{17} \mathrm{~cm}^{-3}$ and responds consequently to the short relaxation time of $\mathrm{PhC} \tau \cong$ $2 \cdot 10^{-10} \mathrm{~s}$. The fact of increasing the $\mathrm{Cr}$ concentration does not simultaneously lead to the relaxation time 


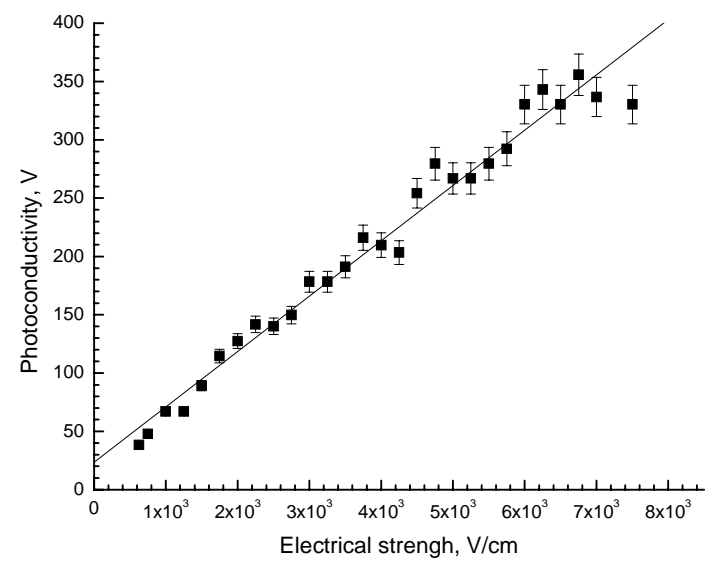

Fig. 3. Dependence of GaAs:Cr photodetector signal magnitude on voltage applied.

decrease. The latter is caused by self-compensation of GaAs:Cr, which is specified by the system of energy levels in the bandgap. As a result of enlargement of the electron capture cross-section on deeper levels (placed close to the medium of the bandgap) that appear after doping, the probability of electron sticking on shallow centers, responsible for nonstationary drop of $\mathrm{PhC}$, decreases but hole sticking still remains, and that is proved by the long-term component of $\mathrm{PhC}$ relaxation drop with the temperature decrease down to $150 \mathrm{~K}$. Taking into account the fact that the time resolution of semi-insulating GaAs photoresistivity detectors is determined by the lifetime of nonstationary electrons:

$$
\tau=\frac{1}{S V N^{0}},
$$

where $N^{0}=N-N^{-}$is the quantity of free sites in impurity centers that can capture electrons, $S$ is the capture cross-section of electrons by impurity center, $V$ is the electron velocity. As one can see [3], $N^{0}$ depends not only on the impurity concentration $N$ but on the degree of the dopant concentration, too. In the case of compensation centers with the concentration $N_{\text {comp }}$, when almost linear growth together with the dopant concentration $N_{\mathrm{Cr}}$ takes place, the following rate dependence $1 / \tau \sim N_{\mathrm{Cr}}$ [6] exists. In the case of pure compensation and $N-N \approx N \approx N^{0}$, when $N$ is close to the degree of $\mathrm{Cr}$ solubility in $\mathrm{GaAs}$, the lifetime can reach a few picoseconds. The detail mechanism of the compensation effect influence on nonstationary component of $\mathrm{PhC}$ has not yet understood and requires special investigations.

The linear dependence of the PhC signal magnitude on electric field applied (Fig. 3) testify to the possibility to increase the detector photosensitivity, if it is necessary up to the electric breakdown threshold $E_{\text {break }}$, $E_{\text {break }} \leq 30 \mathrm{kV} / \mathrm{cm}$ (for some samples the value of $E_{\text {break }}$ was $50 \mathrm{kV} / \mathrm{cm}$ ).

Our investigation of the stationary photoconductivity magnitude $\Delta v_{\mathrm{PhC}}$ dependence on the laser radiation intensity $I$ showed a linear growth of $\Delta v_{\mathrm{PhC}}$ in the range of six orders of $I$ variation (GaAs:Cr samples with $N_{\mathrm{Cr}} \geq$ $\left.1 \cdot 10^{17} \mathrm{~cm}^{-3}\right)$. Nonstationary component of $\mathrm{PhC}$ approached to saturation for maximal $I$. An optimal ratio of fast and slow components (10:1) and photosensitivity was obtained when $N_{\mathrm{Cr}} \cong 3 \cdot 10^{17} \mathrm{~cm}^{-3}$ at the pulse laser intensity starting with $I \sim 3 \cdot 10^{22} \mathrm{~cm}^{-2} \mathrm{~s}^{-1}$ until $1 \cdot 10^{26} \mathrm{~cm}^{-2} \mathrm{~s}^{-1}$ ( $\left.t_{p}=37 \mathrm{ps}, \lambda=1.06 \mu \mathrm{m}\right)$.

\section{Conclusions}

- It was found that, in semi-insulating GaAs:Cr, $N_{\mathrm{Cr}}=3 \cdot 10^{17} \mathrm{~cm}^{-3}$ ensure the relaxation time of photoconductivity $\tau \sim 2 \cdot 10^{-10} \mathrm{~s}$ that corresponds to the bandwidth of the registration system $\Delta f=5 \mathrm{GHz}$ and is optimal for fast detector development with a corresponding time of detection.

- We found a linear growth of the fast component with the intensity increase and approachment to saturation of the slow component at high levels of excitation, which is connected with a full recharge of sticking centers at the laser intensity increase as well as growth of the chromium dopant concentration up to $10^{15} \ldots 3 \cdot 10^{17} \mathrm{~cm}^{-3}$.

- The possibility to control the behavior of $\mathrm{PhC}$ relaxation components in GaAs: $\mathrm{Cr}$ crystals by adjusting degree of doping and compensation has been shown.

\section{References}

1. N. Lovergine, P. Prete, L. Tapfer, F. Marzo, and A.M. Mancini, Hydrogen transport vapour growth and properties of thick CdTe epilayers for RT Xray detector applications // Cryst. Res. Technol. 40 (10-11), p. 1018 - 1022 (2005).

2. J.R. Macril, P. Dufour, L.A. Hamel, M. Julien, M.L. McConnell, M. McClish, J.M. Ryan and M. Widholm, Study of 5 and $10 \mathrm{~mm}$ thick CZT strip detectors // IEEE Nuclear Science Symposium Conference Record, p. 2316-2320 (2001).

3. A. Friant, C. Salou, R. Galli, S. Barday, Picosecond GaAs X- and gamma-ray photodetectors // Nucl. Instrum. and Meth. Phys. Res. A-283, p. 318-322 (1989).

4. D.R. Kania, R.G.Bartlett, R.S. Wagner, R.B. Hammond and P. Pianetta, Fast photoconductors for synchrotron radiation research // Nucl. Instrum. and Meth. Phys. Res. A 222 (1-2), p. 270-273 (1984).

5. Y.J. Chui, S.B. Fleischer, J.E .Bowers, High-speed low temperature-grown GaAs p-i-n traveling-wave photodetector // IEEE Photonics Technology Lett. 10 (7), p. 1012-1014 (1998).

6. Yu.A. Grigoriev, S.P. Grishina, K.P. Konin, V.B. Osvensky, L.F. Linnik, L.G. Linnik, E.A. Salkov, Investigation of recombination process at large level of injection in semi-insulating "unalloyed" gallium arsenide // Kvantovaya elektronika 29, p.79-82 (1985) (in Russian). 